\title{
Extended Gravity: Theory and Phenomenology
}

\section{Salvatore Capozziello*}

Dipartimento di Scienze Fisiche and INFN Sez. di Napoli, Università di Napoli "Federico II", Compl. Univ. di Monte S. Angelo, Edificio G, Via Cinthia, 80121 - Napoli, Italy

E-mail: capozziello@na.infn.it

Extended Theories of Gravity can be related to several quantum gravity approaches and unification schemes. They have recently attracted a lot of interest as alternative candidates to explain the observed cosmic acceleration, the flatness of the rotation curves of spiral galaxies, the gravitational potential of galaxy clusters, and other relevant astrophysical phenomena. Very likely, what we call "Dark Matter" and "Dark Energy" are nothing else but signals of the breakdown of General Relativity at large scales. Furthermore, PPN-parameters deduced from Solar System experiments do not exclude, a priori, the possibility that such theories could give small observable effects also at these scales. We review these results giving the basic ingredients of such an approach.

From Quantum to Emergent Gravity: Theory and Phenomenology

June 11-15 2007

Trieste, Italy

${ }^{*}$ Speaker. 
The impressive amount of good quality data of last decade has shed new light on the effective picture of the Universe. Type Ia Supernovae (SNeIa), anisotropies in the CMBR, and matter power spectrum inferred from large galaxy surveys represent the strongest evidences for a radical revision of the Cosmological Standard Model. In particular, the concordance $\Lambda$ CDM model predicts that baryons contribute only $\sim 4 \%$ of the total matter - energy budget, while the exotic Cold Dark Matter $(\mathrm{CDM})$ represents the bulk of the matter content $(\sim 25 \%)$ and the cosmological constant $\Lambda$ plays the role of the so called Dark Energy $(\sim 70 \%)$.

Although being the best fit to a wide range of data, the $\Lambda$ CDM model is affected by strong theoretical shortcomings that have motivated the search for alternative models [1]. Dark Energy models mainly rely on the implicit assumption that Einstein's General Relativity (GR) is the correct theory of gravity.

Nevertheless, its validity on the larger astrophysical and cosmological scales has never been tested, and it is therefore conceivable that both cosmic speed up and Dark Matter represent signals of a breakdown in our understanding of the gravitational interaction. Following this line of thinking, the choice of a generic function $f(R)$ as the gravitational Lagrangian, where $R$ is the Ricci scalar, can be derived by matching the data and by the "economic" requirement that no exotic ingredients have to be added. This is the underlying philosophy of what are referred to as $f(R)$ gravity [2]. From a theoretical standpoint, different issues suggest that higher order terms must necessarily enter the gravity Lagrangian. In fact, such terms come out as one - loop corrections in field quantization on curved spacetimes and they seem unescapable in any perturbation approach to achieve a self - consistent theory of quantum gravity [3,4].

It is worth noting that Solar System experiments show the validity of GR at these scales so that $f(R)$ theories should not differ too much from GR at this level [5]. In other words, the PPN limit of such models must not violate the experimental constraints on Eddington parameters. A positive answer to this request has been recently achieved for several $f(R)$ theories [6], nevertheless it has to be remarked that this debate is far to be definitively concluded. Although higher order gravity theories have received much attention in cosmology, since they are naturally able to give rise to the accelerating expansion (both in the late [8] and in the early [9] universe), it is possible to demonstrate that $f(R)$ theories can also play a major role at astrophysical scales [10]. In fact, modifying the gravity action can affect the gravitational potential in the low energy limit.

Provided that the modified potential reduces to the Newtonian one on the Solar System scale, this implication could represent an intriguing opportunity rather than a shortcoming for $f(R)$ theories. In fact, a corrected gravitational potential could offer the possibiloty to fit galaxy rotation curves without the need of Dark Matter. In addition, one could work out a formal analogy between the corrections to the Newtonian potential and the usually adopted Dark Matter models. In order to investigate the consequences of $f(R)$ theories on both cosmological and astrophysical scales, let us first remind the basics of this approach.

From a mathematical viewpoint, $f(R)$ theories generalize the Hilbert - Einstein Lagrangian $\mathscr{L}_{H E}=\sqrt{-g} R$ as $\mathscr{L}=\sqrt{-g} f(R)$ without assuming a priori the functional form of Lagrangian density in the Ricci scalar. The field equations are obtained by varying with respect to the metric components to get [2] :

$$
f^{\prime}(R) R_{\alpha \beta}-\frac{1}{2} f(R) g_{\alpha \beta}=f^{\prime}(R)^{; \mu v}\left(g_{\alpha \mu} g_{\beta \nu}-g_{\alpha \beta} g_{\mu v}\right)+T_{\alpha \beta}^{M}
$$


where the prime denotes derivative with respect to the argument and $T_{\alpha \beta}^{M}$ is the standard matter stress - energy tensor. Defining the curvature stress - energy tensor as

$$
T_{\alpha \beta}^{c u r v}=\frac{1}{f^{\prime}(R)}\left\{\frac{1}{6} g_{\alpha \beta}\left[f(R)-R f^{\prime}(R)\right]+f^{\prime}(R)^{; \mu v}\left(g_{\alpha \mu} g_{\beta v}-g_{\alpha \beta} g_{\mu v}\right)\right\} .
$$

Eqs.(1) may be recast in the Einstein - like form as :

$$
G_{\alpha \beta}=R_{\alpha \beta}-\frac{1}{2} g_{\alpha \beta} R=T_{\alpha \beta}^{c u r v}+T_{\alpha \beta}^{M} / f^{\prime}(R)
$$

where matter non - minimally couples to geometry through the term $1 / f^{\prime}(R)$. The presence of term $f^{\prime}(R)_{; \mu \nu}$ renders the equations of fourth order, while, for $f(R)=R$, the curvature stress - energy tensor $T_{\alpha \beta}^{c u r v}$ identically vanishes and Eqs.(3) reduce to the standard second - order Einstein field equations. As it is clear, from Eq.(3), the curvature stress - energy tensor formally plays the role of a further source term in the field equations so that its effect is the same as that of an effective fluid of purely geometrical origin.

However the metric variation is just one of the approaches towards $f(R)$ gravity: in fact, one can face the problem also considering the so called Palatini approach (e.g. see [11, 12]) where the metric and connection fields are considered independent. Apart from some differences in the interpretation, one can deal with a fluid of geometric origin in these case as well.

The scheme outlined above provides all the ingredients we need to tackle with the dark side of the Universe. Depending on the scales, such a curvature fluid can play the role of Dark Matter and Dark Energy. From the cosmological point of view, in the standard framework of a spatially flat homogenous and isotropic Universe, the cosmological dynamics is determined by its energy budget through the Friedmann equations. In particular, the cosmic acceleration is achieved when the r.h.s. of the acceleration equation remains positive (in physical units with $8 \pi G=c=1$ ):

$$
\frac{\ddot{a}}{a}=-\frac{1}{6}\left(\rho_{t o t}+3 p_{t o t}\right),
$$

where $a$ is the scale factor, $H=\dot{a} / a$ the Hubble parameter, the dot denotes derivative with respect to cosmic time, and the subscript tot denotes the sum of the curvature fluid and the matter contribution to the energy density and pressure. From the above relation, the acceleration condition, for a dust dominated model, leads to :

$$
\rho_{\text {curv }}+\rho_{M}+3 p_{\text {curv }}<0 \rightarrow w_{\text {curv }}<-\frac{\rho_{\text {tot }}}{3 \rho_{\text {curv }}}
$$

so that a key role is played by the effective quantities:

$$
\rho_{\text {curv }}=\frac{8}{f^{\prime}(R)}\left\{\frac{1}{2}\left[f(R)-R f^{\prime}(R)\right]-3 H \dot{R} f^{\prime \prime}(R)\right\},
$$

and

$$
w_{\text {curv }}=-1+\frac{\ddot{R} f^{\prime \prime}(R)+\dot{R}\left[\dot{R} f^{\prime \prime \prime}(R)-H f^{\prime \prime}(R)\right]}{\left[f(R)-R f^{\prime}(R)\right] / 2-3 H \dot{R} f^{\prime \prime}(R)} .
$$

As a first simple choice, one may neglect ordinary matter and assume a power-law form $f(R)=$ $f_{0} R^{n}$, with $n$ a real number, which represents a straightforward generalization of the Einstein GR in 


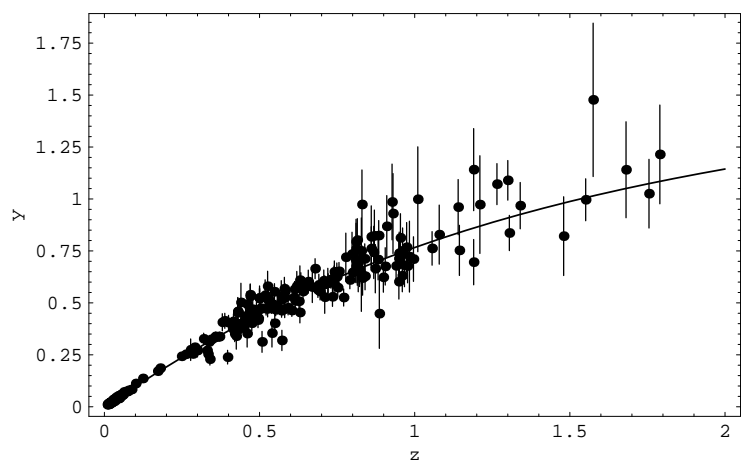

Figure 1: The Hubble diagram of 20 radio galaxies together with the "gold" sample of SNeIa, in term of the redshift as suggested in [13]. The best fit curve refers to the $f(R)$ - gravity model without Dark Matter.

the limit $n=1$. One can find power - law solutions for $a(t)$ providing a satisfactory fit to the SNeIa data and a good agreement with the estimated age of the Universe in the range $1.366<n<1.376$ [7]. On the other side, one can develop the same analysis in presence of the ordinary matter component, although in such a case, one has to numerically solve field equations. Then, it is still possible to confront the Hubble flow described by such a model with the Hubble diagram of SNeIa. The data fit turns out to be significant (see Fig. 1) improving the $\chi^{2}$ value and, it fixes the best fit value at $n=3.46$ when it is accounted only the baryon contribute $\Omega_{b} \approx 0.04$ (according with BBN prescriptions). It has to be remarked that considering Dark Matter does not modify the result of the fit, supporting the assumption of no need for Dark Matter in this model. From the evolution of the Hubble parameter in term of redshift one can even calculate the Age of Universe. The best fit value $n=3.46$ provides $t_{\text {univ }} \approx 12.41 \mathrm{Gyr}$. It is worth noting that considering $f(R)=f_{0} R^{n}$ gravity represents only the simplest generalization of Einstein theory.

In other words, it has to be considered that $R^{n}$ - gravity represents just a working hypothesis as there is no overconfidence that such a model is the correct final gravity theory. In a sense, we want only to suggest that several cosmological and astrophysical results can be well interpreted in the realm of a power law extended gravity model. As matter of fact, this approach gives no rigidity about the value of the power $n$, although it would be preferable to determine a model capable of working at different scales. Furthermore, we do not expect to be able to reproduce the whole cosmological phenomenology by means of a simple power law model, which has been demonstrated to be not sufficiently versatile [14].

For example, we can easily demonstrate that this model fails when it is analyzed with respect to its capability of providing the correct evolutionary conditions for the perturbation spectra of matter overdensity [15]. This point is typically addressed as one of the most important issues which suggest the need for Dark Matter. In fact, if one wants to discard this component, it is crucial to match the observational results related to the Large Scale Structure of the Universe and the Cosmic Microwave Background which show, respectively at late time and at early time, the signature of the initial matter spectrum. As important remark, we note that the quantum spectrum of primordial perturbations, which provides the seeds of matter perturbations, can be positively recovered in the framework of $R^{n}$ - gravity. In fact, $f(R) \propto R^{2}$ can represent a viable model with respect to CMBR data and it is a good candidate for cosmological Inflation. To develop the matter power spectrum 

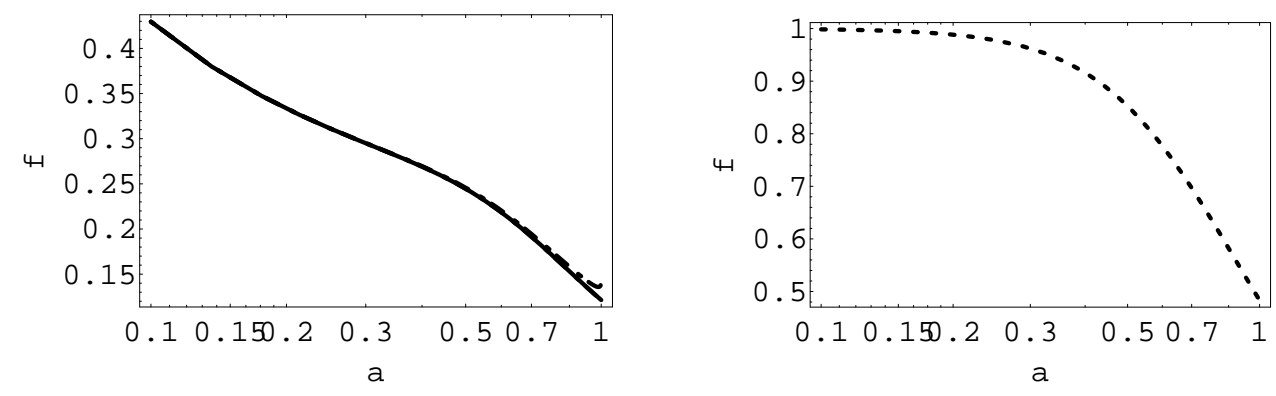

Figure 2: Scale factor evolution of the growth index $f$ : (left) modified gravity, in the case $\Omega_{m}=\Omega_{b a r} \sim$ 0.04 , for the SNeIa best fit model with $n=3.46$, (right) the same evolution in the case of a $\Lambda$ CDM model. In the case of $R^{n}$-gravity it is shown also the dependence on the scale $k$. The three cases $k=0.01,0.001,0.0002$ have been checked. Only the latter case shows a very small deviation from the leading behavior.

suggested by this model, we resort to the equation for the matter contrast obtained in [15] in the case of fourth order gravity. This equation can be deduced considering the conformal Newtonian gauge for the perturbed metric [15]:

$$
d s^{2}=(1+2 \psi) d t^{2}-a^{2}(1+2 \phi) \Sigma_{i=1}^{3}\left(d x^{i}\right) .
$$

In GR, it is $\phi=-\psi$, since there is no anisotropic stress; in extended gravity, this relation breaks, in general, and the $i \neq j$ components of field equations give new relations between $\phi$ and $\psi$. In particular, for $f(R)$ gravity, due to nonvanishing $f_{R ; i ; j}$ (with $i \neq j$ ), the $\phi-\psi$ relation becomes scale dependent. Instead of the perturbation equation for the matter contrast $\delta$, we provide here its evolution in term of the growth index $f=d \ln \delta / d \ln a$, that is the directly measured quantity at $z \sim 0.15:$

$$
f^{\prime}(a)-\frac{f(a)^{2}}{a}+\left[\frac{2}{a}+\frac{1}{a} E^{\prime}(a)\right] f(a)-\frac{1-2 Q}{2-3 Q} \cdot \frac{3 \Omega_{m} a^{-4}}{n E(a)^{2} \tilde{R}^{n-1}}=0,
$$

$E(a)=H(a) / H_{0}, \tilde{R}$ is the dimensionless Ricci scalar, and

$$
Q=-\frac{2 f_{R R} c^{2} k^{2}}{f_{R} a^{2}}
$$

For $n=1$ the previous expression gives the ordinary growth index relation for the Cosmological Standard Model. It is clear, from Eq.(9), that such a model suggests a scale dependence of the growth index which is contained into the corrective term $Q$ so that, when $Q \rightarrow 0$, this dependence can be reasonably neglected. In the most general case, one can resort to the limit $a H<k<$ $10^{-3} h \mathrm{Mpc}^{-1}$, where Eq.(9) is a good approximation, and non-linear effects on the matter power spectrum can be neglected.

Studying numerically Eq.(9), one obtains the growth index evolution in term of the scale factor; for the sake of simplicity, we assume the initial condition $f\left(a_{l s}\right)=1$ at the last scattering surface as in the case of matter-like domination. The results are summarized in Fig.(2), where we show, in parallel, the growth index evolution in $R^{n}$ - gravity and in the $\Lambda$ CDM model.

In the case of $\Omega_{m}=\Omega_{b a r} \sim 0.04$, one can observe a strong disagreement between the expected rate of the growth index and the behavior induced by power law fourth order gravity models. This 
negative result is evidenced by the predicted value of $f\left(a_{z=0.15}\right)$, which has been observationally estimated by the analysis of the correlation function for 220000 galaxies in 2dFGRS dataset sample at the survey effective depth $z=0.15$. The observational result suggests $f=0.58 \pm 0.11$ [16], while our model gives $f\left(a_{z=0.15}\right) \sim 0.117(k=0.01), 0.117(k=0.001), 0.122(k=0.0002)$. Although this result seems frustrating with respect to the underlying idea to discard the dark components from the cosmological dynamics, it does not give substantial improvement in the case of $R^{n}$ gravity model plus Dark Matter. In fact, it is possible to show that, even in this case, the growth index prediction is far to be in agreement with the $\Lambda \mathrm{CDM}$ model and again, at the observational scale $z=0.15$, there is not enough growth of perturbations to match the observed Large Scale Structure. In such a case one obtains : $f\left(a_{z=0.15}\right) \sim 0.29(k=0.01), 0.29(k=0.001), 0.31(k=0.0002)$, which are quite increased with respect to the previous case but still very far from the experimental estimate. It is worth noting that no significantly different results are obtained if one varies the power $n$, of course in the case of $n \rightarrow 1$ one recovers the standard behavior if a cosmological constant contribution is added. These results seem to suggest that an extended gravity model which considers a simple power law of Ricci scalar, although cosmologically relevant at late times, is not viable to describe the evolution of Universe at all scales. In other words, such a scheme seems too simple to give account for the whole cosmological phenomenology. In fact, in [15] a gravity Lagrangian considering an exponential correction to the Ricci scalar $f(R)=R+A \exp (-B R)$ (with $A, B$ two constants), gives more interesting results and displays a grow factor rate which is in agreement with the observational results at least in the Dark Matter case. To corroborate this point of view, one has to consider that when the choice of $f(R)$ is performed starting from observational data (pursuing an inverse approach) as in [17], the reconstructed Lagrangian is a non - trivial polynomial in term of the Ricci scalar. A result which directly suggests that the whole cosmological phenomenology can be accounted only with a suitable non - trivial function of the Ricci scalar rather than a simple power law function. As matter of fact, the results obtained with respect to the study of the matter power spectra in the case of $R^{n}$ - gravity do not invalidate the whole approach, since they can be referred to the too simple form of the model.

The results obtained at cosmological scales motivates further analysis of $f(R)$ theories. In a sense, one is wondering whether the curvature fluid, which works as Dark Energy, can also play the role of effective Dark Matter thus yielding the possibility of recovering the observed astrophysical phenomenology by the only visible matter. It is well known that, in the low energy limit, higher order gravity implies a modified gravitational potential. Therefore, in our discussion, a fundamental role is played by the new gravitational potential descending from the given fourth order gravity theories we are referring to. By considering the case of a pointlike mass $m$ and solving the vacuum field equations for a Schwarzschild - like metric, one gets from a theory $f(R)=f_{0} R^{n}$, the modified gravitational potential [10]:

$$
\Phi(r)=-\frac{G m}{2 r}\left[1+\left(\frac{r}{r_{c}}\right)^{\beta}\right]
$$

where

$$
\beta=\frac{12 n^{2}-7 n-1-\sqrt{36 n^{4}+12 n^{3}-83 n^{2}+50 n+1}}{6 n^{2}-4 n+2}
$$

which corrects the ordinary Newtonian potential by a power - law term. In particular, this correction sets in on scales larger than $r_{c}$ which value depends essentially on the mass of the system. The 
corrected potential (11) reduces to the standard $\Phi \propto 1 / r$ for $n=1$ as it can be seen from the relation (12).

The result (11) deserves some comments. As discussed in detail in [10], we have assumed the spherically symmetric metric and imposed it into the field equations (1) considered in the weak field limit approximation. As a result, we obtain a corrected Newtonian potential which accounts for the strong non-linearity of gravity related to the higher-order theory. However, we have to notice that Birkhoff's theorem does not hold, in general, for $f(R)$ gravity (see [18] for a demonstration) but other spherically symmetric solutions than the Schwarzschild one can be found in these extended theories of gravity [19].

The generalization of Eq.(11) to extended systems is achieved by dividing the system in infinitesimal mass elements and summing up the potentials generated by each single element. In the continuum limit, we replace the sum with an integral over the mass density of system taking care of eventual symmetries of the mass distribution (see [10] for details). Once the gravitational potential has been computed, one may evaluate the rotation curve $v_{c}^{2}(r)$ and compare it with the data. For extended systems, one has typically to resort to numerical techniques, but the main effect may be illustrated by the rotation curve for the pointlike case, that is:

$$
v_{c}^{2}(r)=\frac{G m}{2 r}\left[1+(1-\beta)\left(\frac{r}{r_{c}}\right)^{\beta}\right] .
$$

Compared with the Newtonian result $v_{c}^{2}=G m / r$, the corrected rotation curve is modified by the addition of the second term in the r.h.s. of Eq.(13). For $0<\beta<1$, the corrected rotation curve is higher than the Newtonian one. Since measurements of spiral galaxies rotation curves signals a circular velocity higher than those which are predicted on the basis of the observed luminous mass and the Newtonian potential, the above result suggests the possibility that our modified gravitational potential may fill the gap between theory and observations without the need of additional Dark Matter.

It is worth noting that the corrected rotation curve is asymptotically vanishing as in the Newtonian case, while it is usually claimed that observed rotation curves are flat (i.e., asymptotically constant). Actually, observations do not probe $v_{c}$ up to infinity, but only show that the rotation curve is flat within the measurement uncertainties up to the last measured point. This fact by no way excludes the possibility that $v_{c}$ goes to zero at infinity. In order to observationally check the above result, we have considered a sample of LSB galaxies with well measured $\mathrm{HI}+\mathrm{H} \alpha$ rotation curves extending far beyond the visible edge of the system. LSB galaxies are known to be ideal candidates to test Dark Matter models since, because of their high gas content, the rotation curves can be well measured and corrected for possible systematic errors by comparing $21-\mathrm{cm} \mathrm{HI} \mathrm{line}$ emission with optical $\mathrm{H} \alpha$ and [NII] data. Moreover, they are supposed to be Dark Matter dominated so that fitting their rotation curves without this elusive component is a strong evidence in favor of any successful alternative theory of gravity.

Our sample contains 15 LSB galaxies with data on both the rotation curve, the surface mass density of the gas component and $R$ - band disk photometry extracted from a larger sample selected by de Blok \& Bosma [20]. We assume the stars are distributed in an infinitely thin and circularly symmetric disk with surface density $\Sigma(r)=\Upsilon_{\star} I_{0} \exp \left(-r / r_{d}\right)$ where the central surface luminosity 

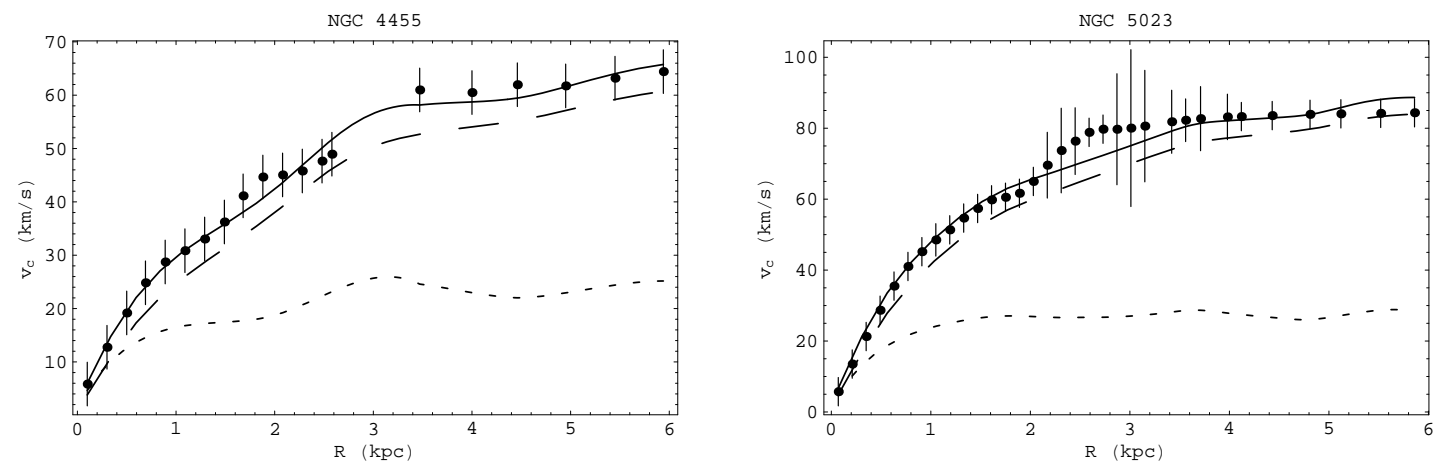

Figure 3: Best fit theoretical rotation curve superimposed to the data for the LSB galaxy NGC 4455 (left) and NGC 5023 (right). To better show the effect of the correction to the Newtonian gravitational potential, we report the total rotation curve $v_{c}(r)$ (solid line), the Newtonian one (short dashed) and the corrected term (long dashed).

$I_{0}$ and the disk scalelength $r_{d}$ are obtained from fitting to the stellar photometry. The gas surface density has been obtained by interpolating the data over the range probed by $\mathrm{HI}$ measurements and extrapolated outside this range. When fitting to the theoretical rotation curve, there are three quantities to be determined, namely the stellar mass - to - light (M/L) ratio, $\Upsilon_{\star}$ and the theory parameters $\left(\beta, r_{c}\right)$. It is worth stressing that, while fit results for different galaxies should give the same $\beta, r_{c}$ is related to one of the integration constants of the field equations. As such, it is not a universal quantity and its value must be set on a galaxy - by - galaxy basis. However, it is expected that galaxies having similar properties in terms of mass distribution have similar values of $r_{c}$ so that the scatter in $r_{c}$ must reflect somewhat the scatter in the circular velocities. In order to match the model with the data, we perform a likelihood analysis determining for each galaxy, using, as fitting parameters $\beta, \log r_{c}$ (with $r_{c}$ in kpc) and the gas mass fraction ${ }^{1} f_{g}$. As it is evident considering the results from the different fits, the experimental data are successfully fitted by the model (see [10] for details). In particular, for the best fit range of $\beta(\beta=0.80 \pm 0.08)$, one can conclude that $R^{n}$ gravity with $2.3<n<5.3$ (best fit value $n=3.2$ which well overlaps the above mentioned range of $n$ fitting SNeIa Hubble diagram) can be a good candidate to solve the missing matter problem in LSB galaxies without any Dark Matter.

At this point, it is worth wondering whether a link may be found between $R^{n}$ gravity and the standard approach based on Dark Matter haloes since both theories fit equally well the same data. As a matter of fact, it is possible to define an effective Dark Matter halo by imposing that its rotation curve equals the correction term to the Newtonian curve induced by $R^{n}$ gravity. Mathematically, one can split the total rotation curve derived from $R^{n}$ gravity as $v_{c}^{2}(r)=v_{c, N}^{2}(r)+v_{c, c o r r}^{2}(r)$ where the second term is the correction. Considering, for simplicity a spherical halo embedding a thin exponential disk, we may also write the total rotation curve as $v_{c}^{2}(r)=v_{c, d i s k}^{2}(r)+v_{c, D M}^{2}(r)$ with $v_{c, d i s k}^{2}(r)$ the Newtonian disk rotation curve and $v_{c, D M}^{2}(r)=G M_{D M}(r) / r$ the Dark Matter one,

\footnotetext{
${ }^{1}$ This is related to the $M / L$ ratio as $\Upsilon_{\star}=\left[\left(1-f_{g}\right) M_{g}\right] /\left(f_{g} L_{d}\right)$ with $M_{g}=1.4 M_{H I}$ the gas $(\mathrm{HI}+\mathrm{He})$ mass, $M_{d}=\Upsilon_{\star} L_{d}$ and $L_{d}=2 \pi I_{0} r_{d}^{2}$ the disk total mass and luminosity.
} 
$M_{D M}(r)$ being its mass distribution. Equating the two expressions, we get:

$$
M_{D M}(\eta)=M_{v i r}\left(\frac{\eta}{\eta_{v i r}}\right) \frac{2^{\beta-5} \eta_{c}^{-\beta}(1-\beta) \eta^{\frac{\beta-5}{2}} \mathscr{I}_{0}(\eta)-\mathscr{V}_{d}(\eta)}{2^{\beta-5} \eta_{c}^{-\beta}(1-\beta) \eta^{\frac{\beta-5}{2}} \mathscr{I}_{0}\left(\eta_{v i r}\right)-\mathscr{V}_{d}\left(\eta_{v i r}\right)} .
$$

with $\eta=r / r_{d}, \Sigma_{0}=\Upsilon_{\star} i_{0}, \mathscr{V}_{d}(\eta)=I_{0}(\eta / 2) K_{0}(\eta / 2) \times I_{1}(\eta / 2) K_{1}(\eta / 2)^{2}$ and :

$$
\mathscr{I}_{0}(\eta, \beta)=\int_{0}^{\infty} \mathscr{F}_{0}\left(\eta, \eta^{\prime}, \beta\right) k^{3-\beta} \eta^{\prime \frac{\beta-1}{2}} \mathrm{e}^{-\eta^{\prime}} d \eta^{\prime}
$$

with $\mathscr{F}_{0}$ only depending on the geometry of the system and "vir" indicating virial quantities. Eq.(14) defines the mass profile of an effective spherically symmetric Dark Matter halo whose ordinary rotation curve provides the part of the corrected disk rotation curve due to the addition of the curvature corrective term to the gravitational potential. It is evident that, from an observational viewpoint, there is no way to discriminate between this dark halo model and $R^{n}$ gravity.

Having assumed spherical symmetry for the mass distribution, it is immediate to compute the mass density for the effective dark halo as $\rho_{D M}(r)=\left(1 / 4 \pi r^{2}\right) d M_{D M} / d r$. The most interesting features of the density profile are its asymptotic behaviors that may be quantified by the logarithmic slope $\alpha_{D M}=d \ln \rho_{D M} / d \ln r$ which can be computed only numerically as function of $\eta$ for fixed values of $\beta$ (or $n$ ). As expected, $\alpha_{D M}$ depends explicitly on $\beta$, while $\left(r_{c}, \Sigma_{0}, r_{d}\right)$ enter indirectly through $\eta_{v i r}$. The asymptotic values at the center and at infinity denoted as $\alpha_{0}$ and $\alpha_{\infty}$ result particularly interesting. It turns out that $\alpha_{0}$ almost vanishes so that in the innermost regions the density is approximately constant. Indeed, $\alpha_{0}=0$ is the value corresponding to models having an inner core such as the cored isothermal sphere and the Burkert model [21].

Moreover, it is well known that galactic rotation curves are typically best fitted by cored dark halo models. On the other hand, the outer asymptotic slope is between -3 and -2 , that are values typical of most dark halo models in literature. In particular, for $\beta=0.80$ one finds $\left(\alpha_{0}, \alpha_{\infty}\right)=$ $(-0.002,-2.41)$, which are quite similar to the value for the Burkert model $(0,-3)$. It is worth noting that the Burkert model has been empirically proposed to provide a good fit to the LSB and dwarf galaxies rotation curves. The values of $\left(\alpha_{0}, \alpha_{\infty}\right)$ we find for the best fit effective dark halo therefore suggest a possible theoretical motivation for the Burkert-like models. Due to the construction, the properties of the effective Dark Matter halo are closely related to the disk one. As such, we do expect some correlation between the dark halo and the disk parameters. To this aim, exploiting the relation between the virial mass and the disk parameters, one can obtain a relation for the Newtonian virial velocity $V_{v i r}=G M_{v i r} / r_{v i r}$ :

$$
M_{d} \propto \frac{\left(3 / 4 \pi \delta_{t h} \Omega_{m} \rho_{c r i t}\right)^{\frac{1-\beta}{4}} r_{d}^{\frac{1+\beta}{2}} \eta_{c}^{\beta}}{2^{\beta-6}(1-\beta) G^{\frac{5-\beta}{4}}} \frac{V_{v i r}^{\frac{5-\beta}{2}}}{\mathscr{I}_{0}\left(V_{v i r}, \beta\right)} .
$$

We have numerically checked that Eq.(16) may be well approximated as $M_{d} \propto V_{v i r}^{a}$ which has the same formal structure as the baryonic Tully - Fisher (BTF) relation $M_{b} \propto V_{\text {flat }}^{a}$ with $M_{b}$ the total (gas + stars) baryonic mass and $V_{\text {flat }}$ the circular velocity on the flat part of the observed rotation curve. In order to test whether the BTF can be explained thanks to the effective Dark Matter halo we are proposing, we should look for a relation between $V_{v i r}$ and $V_{\text {flat }}$. This is not analytically

\footnotetext{
${ }^{2}$ Here $I_{l}$ and $K_{l}$, with $l=1,2$ are the Bessel functions of first and second type.
} 
possible since the estimate of $V_{\text {flat }}$ depends on the peculiarities of the observed rotation curve such as how far it extends and the uncertainties on the outermost points. For given values of the disk parameters, we therefore simulate theoretical rotation curves for some values of $r_{c}$ and measure $V_{\text {flat }}$ finally choosing the fiducial value for $r_{c}$ that gives a value of $V_{\text {flat }}$ as similar as possible to the measured one. Inserting the relation thus found between $V_{\text {flat }}$ and $V_{\text {vir }}$ into Eq.(16) and averaging over different simulations, we finally get:

$$
\log M_{b}=(2.88 \pm 0.04) \log V_{\text {flat }}+(4.14 \pm 0.09)
$$

while a direct fit to the observed data gives [22]:

$$
\log M_{b}=(2.98 \pm 0.29) \log V_{\text {flat }}+(3.37 \pm 0.13) .
$$

The slope of the predicted and observed BTF are in good agreement thus leading further support to our approach. The zeropoint is markedly different with the predicted one being significantly larger than the observed one. However, it is worth stressing that both relations fit the data with similar scatter. A discrepancy in the zeropoint can be due to our approximate treatment of the effective halo which does not take into account the gas component. Neglecting this term, we should increase the effective halo mass and hence $V_{v i r}$ which affects the relation with $V_{\text {flat }}$ leading to a higher than observed zeropoint. Indeed, the larger is $M_{g} / M_{d}$, the more the points deviate from our predicted BTF thus confirming our hypothesis. Given this caveat, we can conclude, with confidence, that $R^{n}$ gravity offers a theoretical foundation even for the empirically found BTF relation.

Although the results outlined along this paper are referred to a simple choice of fourth order gravity models $\left(f(R)=f_{0} R^{n}\right)$ they could represent an interesting paradigm. In fact, even if such a model is not suitable to provide the correct form of the matter power spectra, and this suggests that a more complicated Lagrangian is needed to reproduce the whole dark sector phenomenology at all scales, we have shown that considering extensions of GR can allow to explain some important issues of cosmological and astrophysical phenomenology. We have seen that extended gravity models can reproduce SNeIa Hubble diagram without Dark Matter, giving significant predictions even with regard to the age of Universe. In addition, the modification of the gravitational potential which arises as a natural effect in the framework of higher order gravity can represent a fundamental tool to interpret the flatness of rotation curves of LSB galaxies. Furthermore, if one considers the model parameters settled by the fit over the observational data on LSB rotation curves, it is possible to construct a phenomenological analogous of Dark Matter halo whose shape is similar to the one of the Burkert model. Since the Burkert model has been empirically introduced to give account of the Dark Matter distribution in the case of LSB and dwarf galaxies, this result could represent an interesting achievement since it gives a theoretical foundation to such a model.

By investigating the relation among dark halo and the disk parameters, we have deduced a relation between $M_{d}$ and $V_{\text {flat }}$ which reproduces the baryonic Tully - Fisher. In fact, exploiting the relation between the virial mass and the disk parameters, one can obtain a relation for the virial velocity which can be satisfactory approximated as $M_{d} \propto V_{v i r}^{a}$. Even such a result seems very intriguing since it gives again a theoretical interpretation for a phenomenological relation. As a matter of fact, although not definitive, these phenomenological issues on $f(R)$ can represent a viable approach for future investigations and in particular support the quest for a unified view of the dark side of the Universe. 
In summary, these results motivate a careful search for a fundamental theory of gravity able to explain the full cosmic dynamics with the only two main ingredients which we can directly experience, namely the background gravity and the baryonic matter.

\section{References}

[1] Copeland E.J., Sami M., Tsujikawa S. 2006, Int. J. Mod. Phys. D 15, 1753

[2] Capozziello S. 2002, Int. J. Mod. Phys. D, 11, 483

[3] Birrell N.D. and Davies P.C.W. 1982, Quantum Fields in Curved Space, Cambridge Univ. Press, Cambridge

[4] Buchbinder I.L., Odintsov S.D., and Shapiro I.L. 1992, Effective Action in Quantum Gravity, IOP Publishing, Bristol

[5] Olmo G.J. 2005, Phys. Rev. Lett. 95, 261102

[6] Capozziello S., Troisi A. 2005, Phys. Rev. D, 72, 044022

[7] Capozziello S., Cardone V.F., Carloni S., Troisi A. 2003, Int. J. Mod. Phys. D, 12, 1969

[8] Carroll, S.M., Duvvuri, V., Trodden, M., Turner, M.S. 2004, Phys. Rev. D, 70, 043528

[9] Starobinsky A.A. 1980, Phys. Lett. B, 91, 99

[10] S. Capozziello, V.F. Cardone, A. Troisi 2007, Mon. Not. Roy. Astron. Soc. 375, 1423

[11] Magnano G., Ferraris M., and Francaviglia M. 1987, Gen. Rel. Grav. 19, 465

[12] Sotiriou T.P. and Liberati S. 2007, Ann. Phys. 322, 935

[13] Daly R.A., Djorgovsky S.G., 2004, Astrophys. J., 612, 652

[14] Capozziello S., Nojiri S., Odintsov S.D., Troisi A., 2006, Phys. Lett. B (2006) 135.

[15] Zhang P., 2006, Phys. Rev. D, 73, 123504

[16] Lahav O., et al., 2002, MNRAS, 333, 961

[17] Capozziello S., Cardone V.F., Troisi A. 2005, Phys. Rev. D, 71, 043503

[18] Capozziello S., Stabile A., Troisi A. 2007, Phys. Rev. D, in press arXiv:0708.0723

[19] Capozziello S., Stabile A., Troisi A. 2007, Class. Quant. Grav. 24, 2153

[20] de Blok W.J.G., Bosma A. 2002, Astron. Astroph., 385, 816

[21] Burkert A. 1995, Astroph. Journ., 447, L25.

[22] McGaugh S.S. 2005, Astroph. Journ., 632, 859 